\title{
Design and Implementation of Embedded Teaching Experiment based on MSP430
}

\author{
Wu Yongchong \\ Jiangxi University of Technology,Continuing Education Institute
}

\begin{abstract}
With the development of electronic technology, more and more single chip microcomputer and embedded teaching courses are open in electronic specialty of colleges and universities. However, the courses only teach relevant principle or theory without deep content and cannot form a complete system. Concerning this situation, this paper develops embedded teaching experiment platform based on MSP430 single chip microcomputer which has two functional features comparing to similar teaching experiment platforms: the first is sound module, and the other is rich interface resources. This platform takes control technology of traditional single chip microcomputer in terms of internal resources into consideration which also integrates digital signal processing MPA and DMA technology. It is almost equal to high performance embedded system platform such as DSP and ARM, which satisfies basic experimental teaching and can be applied to product development.
\end{abstract}

KeyWords-Electrical Engineering; Embedded System; Single Chip Microcomputer; MSP430

\section{INTRODUCTION}

In the information era with rapid development of science and technology as well as continuous integration, the antagonism of traditional communication and radar system has become a memory. Besides, the electronic warfare under high-tech conditions does not only include communication, radar, optical, camouflage, navigation and guidance systems, extending all over space, air, land, surface and underwater, covering meter-wave, microwave, millimeter-wave and all electromagnetic spectrums, involving various arms of service and operation areas. With the increasing demand for electronics, electronic technology gradually becomes the focus of engineering construction, and China also begins to vigorously develop electronic project construction. However, China has a lagging behind basis which has a huge gap comparing to international electronic engineering. In addition, with the continuous development of economic globalization, electronic engineering in China is facing a lot of challenges.

This thesis completes component selection schematic design and PCB drawing for hardware module's periphery module. Firstly, it sets peripheral resources based on the design requirement of overall scheme and integrated analog/digital of MSP430F169 microcontroller to determine necessary device of input keyboard and display module, real time clock module, data acquisition module, communication module, data storage and file management module; then, we can carry out module schematic design; the final step is to draw PCB. The entire hardware system is compact and the resources distribution on the Board is reasonable.

\section{BACKGROUND AND MEANING FOR EMBEDDED}

\section{TEACHING EXPERIMENT}

In recent years, the continuous updating of information and electronic technology provide necessary condition for universities to carry out opening laboratories and students can innovate their ability and improve various qualities while learning and training in the opening laboratories. A suitable platform is very important to cultivate students' innovative ability.

Embedded teaching experiment platforms have diverse varieties with uneven performance and big price variance from dozens of Yuan to thousands of Yuan. In College and universities, parts of embedded courses are implemented based on DSP, ARM or FPGA with high-tech chip and the teaching structure as well as principle is complicated. However, beginners have to be equipped with strong theoretical knowledge while using, so it is difficult to get started which needs a long period of 
time to develop. Single-chip MCU is similar to DSP and ARM in terms of structure, which is simple from the perspective of principle and application and is entry-level experimental platform for students to understand embedded system. After market research, we find out that those with suitable price for students have simple structure yet few function while those with good performance cost much and are not suitable for promotion.

At present, various colleges and universities offer single-chip microcomputer courses, teaching 51 single-chip microcomputer to 430 single-chip, which take students ' knowledge structure and affordability into consideration. This paper designs and develops MSP430 microcontroller-based embedded teaching experiment platform.

\section{SYSTEM COMPOSITION AND FUNCTION INTRODUCTION}

\section{OF EXPERIMENTAL PLATFORM SYSTEM}

The main chip of teaching experiment platform adopts 6-bit ultra-low power MSP43OF169 microcontroller introduced by T1. It adopts Von Neumann structure, while RAM, ROM and all periphery modules are located within the same space, and the maximum addressing range is 62KB (6oKBFlash, ZKBRAM). Besides, internal integration includes watchdog timer one hardware multiplier, one accurate analog comparators, 2 timers with capture/compare register, 8-channel 12-bit AD converter, 2-channel 12-bit DAC converter, USART serial communications interface supporting IZC and SPI communication protocol and 48 1/0 pins which support DMA data transfers. MSP430F169 is FLASH memory type single-chip which is equipped with JTAG emulation interface and high-level language compilers, supporting emulation debugging. Based on rich internal resources of MSP430, this paper constructs a complete embedded teaching experiment platform with several common modules. The experimental platform module construction is shown inFig.1.

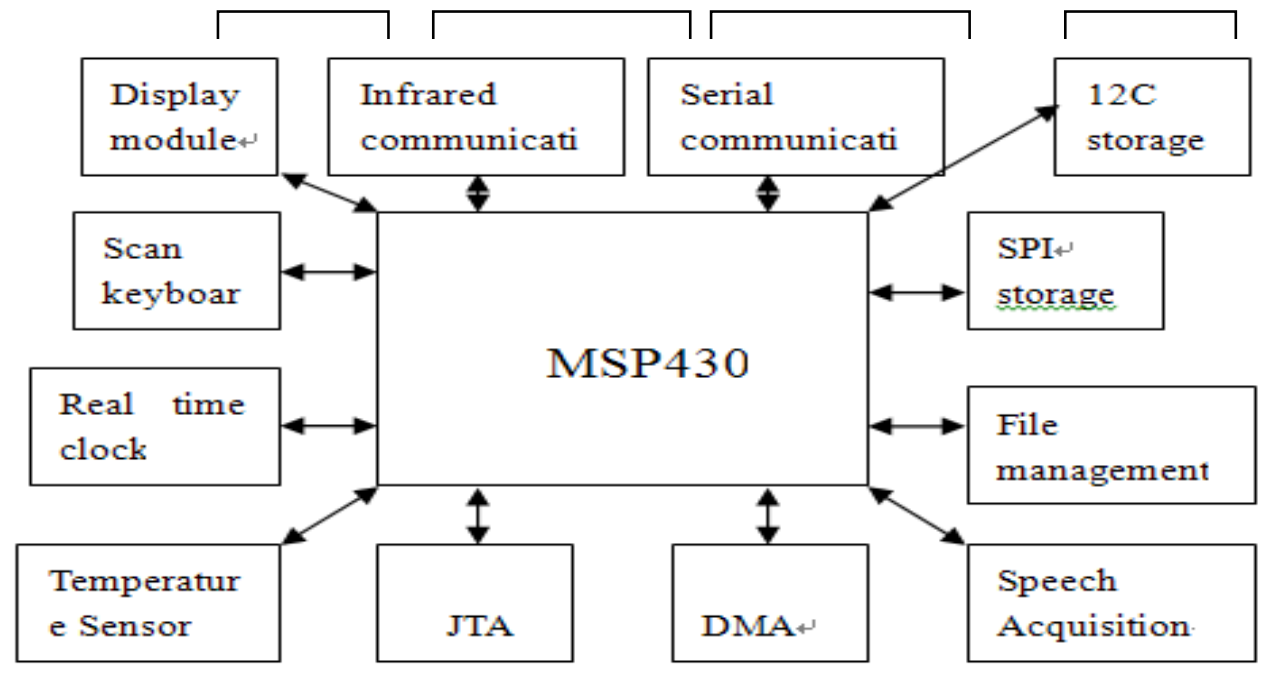

Figure 1.

This experimental platform includes basic module and extension module and the basic module mainly includes:

LED display and seven segment display demonstration module

Scan keyboard module

Real time clock module

Unibus DSISBZO temperature module

$\mathrm{RS}$-232 communication module

Infrared wireless communication module

IZC serial storage module
SPI and serial storage module

Top knife D and D/A application module

SD card and USBfile management module etc

MSP43O microcontroller's characteristics

The MSP430 ultra-low power 16-bit mixed-signal processors produced by American Texas Instruments integrates various advanced technologies to define the concept for a new-generation microcontroller based on 16-bit RISC processor, high-performance analog technology, ultra low power consumption, rich on-chip 
peripherals, JTAG emulation and debugging. MSP430 microcontroller includes five products, each product provides varying levels of analog integration, digital peripherals and communication protocols. The internal structure for MSP43O is shown in Fig .2:

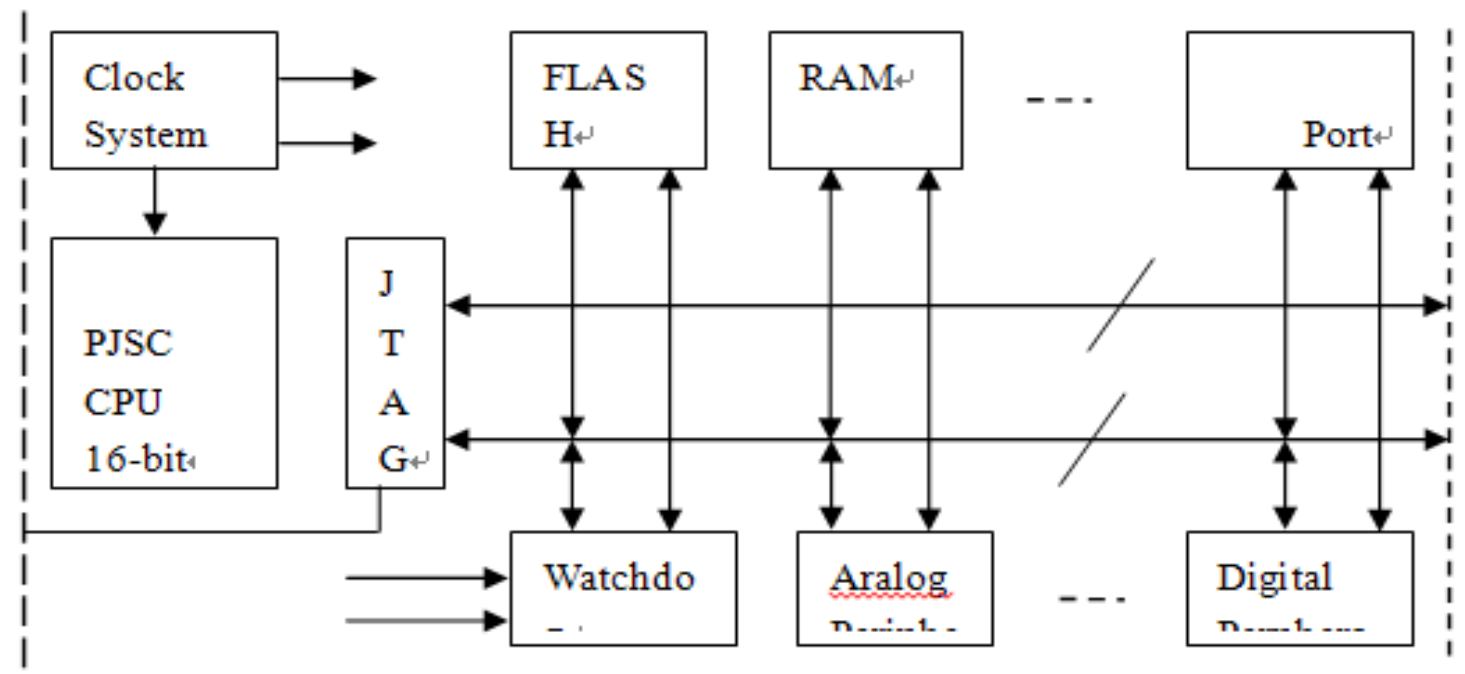

Figure 2. MSP430 internal structure

MsP430 combines advanced CPU module and modular memory map peripherals to provide solutions for present and future mixed-signal applications. Its characteristics can be summarized as follows:

\section{A. Strong operational performance}

In terms of operational performance, the MCU of MSP430 microcontroller adopts 16-bit reduced instruction set computer architecture, providing 16 highly flexible single-cycle 16-bit CPU registers operation. The CPU of this design is simple with rich functions which adopts 27 simple operation orders and 7 uniform addressing mode and can efficiently carry out computing with smaller size and higher code rates.

\section{B. Flexible clock system}

MSP43OMCU clock system is specially designed for battery-powered applications. Various oscillators can be used to support event-driven tasks. Besides, ACLK is able to drive based on internal ultra low-power oscillator without additional external components. ACLK is available as your backup real time clock to remind waking up.

\section{Ultra low power design}

MSP430 is carefully designed for low power applications. Highly flexible clock system, a variety of operating modes and zero power consumption BOR (zero-power always on BoR) cannot only greatly reduce power consumption, but also significantly extend the battery life. MPS430BOR function will always be in working condition, and reliable performance can be guaranteed in low-power mode.

\section{CONCLUSION}

This paper mainly completes the design and implementation of embedded teaching experiment platform based on MSP430 microcontroller which has two functional features comparing to similar teaching experiment platforms: the first is sound module, and the other is rich interface resources. This platform takes control technology of traditional single chip microcomputer in terms of internal resources into consideration which also integrates digital signal processing MPA and DMA technology. It is almost equal to high performance embedded system platform such as DSP and ARM, which satisfies basic experimental teaching and can be applied to product development. This paper mainly completes the following tasks:

It completes overall teaching experiment platform project demonstration. First of all, it carries out comparison and analysis on the comparison and performance of common single-chip computers in detail and then, it compares MSP430 MCU and integrated 
hardware resources of DSP and ARM to finally choose MSP430F169 microcontroller as the main chip of this experiment. In addition, it combines rich interface resources and characteristics of MSP430F169 to determine the whole frame structure of experimental platform.

\section{REFERENCES}

[1] Yamagami, Y., Nishio, Y., Kawata, J., \& Ushida, A. (2004). $\dagger$ department of electrical and electronic engineering,. Harbo, 6(6), 377-390.

[2] Warden, P. W. "Electronic engineering in oceanography." Radio \& Electronic Engineer 32(1966).

[3] Hu J, Li Y K, Nie Z, et al. School of Electronic Engineering, University of Electronic Science and Technology of China, Chengdu, China[J]. Antennas \& Propagation IEEE Transactions on, 2014, 62(19):870-877.

[4] Colace F, De Santo M, Pietrosanto A. Work in progress - virtual lab for electronic engineering curricula[C]// 2013 IEEE Frontiers in Education Conference (FIE). IEEE, 2004:T3C/22-T3C/24 Vol.
[5] Condon, Marissa, Deaño, Alfredoand Iserles, Arieh, , Deaño, Alfredo, and Iserles, Arieh. On highly oscillatory problems arising in electronic engineering $[\mathrm{J}]$. Esaim Mathematical Modelling \& Numerical Analysis, 2009, 43(4):785-804.

[6] Cobby M, Nicol D, Durrani T S, et al. Teaching electronic engineering via the World Wide Web[C]// Computer Based Learning in Electronic Education, IEE Colloquium on. IET, 1995:7/1 - 711

[7] Abbott, Paul. "Integrated electrical and electronic engineering for mechanical engineers." Assembly Automation 1(1994).

[8] Liao, W., Gao, G., Wang, Y., Tian, W., Rui, G., \& Fu, Z., et al. (2012). Advances in Electronic Engineering, Communication and Management Vol.2. Springer Berlin Heidelberg.

[9] C. T. Medrano, M. Ubé, I. Plaza, \& A. Blesa. (2002). The tools of quality in electronic engineering education. European Journal of Engineering Education, 27(4), 325-337.

[10] Ma, Can. "CFRP Application in Electronic Engineering." HI-TECH FIBER \& APPLICATION (1999).

[11] Liang, L., \& Li, C. (2009). Reformation to practical teaching in opto-electronic engineering. Journal of Science of Teachers College \& University.

[12] Medrano, C., Plaza, I., Castro, M., García-Sevilla, F., Martínez-Calero, J. D., \& Felix, J. P., et al. (2010). A review of electronic engineering design free software tools. Education Engineering (EDUCON), 2010 IEEE (pp.1867-1871). IEEE. 